\title{
Wave-induced vortex generation around a slender vertical cylinder ${ }^{\circledR}$
}

Cite as: Phys. Fluids 32, 042105 (2020); https://doi.org/10.1063/1.5141131

Submitted: 06 December 2019. Accepted: 07 April 2020 . Published Online: 24 April 2020

Giulia Antolloni, (D) Atle Jensen, (D) John Grue, Bjørn H. Riise, and (D) Maurizio Brocchini

\section{COLLECTIONS}

EP This paper was selected as an Editor's Pick
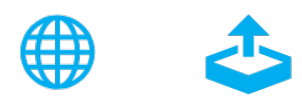

\section{ARTICLES YOU MAY BE INTERESTED IN}

Flow separation around a square cylinder at low to moderate Reynolds numbers

Physics of Fluids 32, 044103 (2020); https://doi.org/10.1063/5.0005757

Vortex shedding characteristics in the wake of circular finned cylinders

Physics of Fluids 32, 045113 (2020); https://doi.org/10.1063/5.0005079

Aerodynamic performance of a free-flying dragonfly-A span-resolved investigation

Physics of Fluids 32, 041903 (2020); https://doi.org/10.1063/1.5145199

\section{Physics of Fluids}

SPECIAL TOPIC: Tribute to

Frank $M$. White on his 88th Anniversary 


\title{
Wave-induced vortex generation around a slender vertical cylinder ${ }^{[}$?
}

\author{
Cite as: Phys. Fluids 32, 042105 (2020); doi: 10.1063/1.5141131 \\ Submitted: 6 December 2019 • Accepted: 7 April 2020 • \\ Published Online: 24 April 2020
}

Giulia Antolloni, ${ }^{7}$ Atle Jensen, ${ }^{2, a)}$ (D) John Grue, ${ }^{2}$ (D) Bjørn H. Riise, ${ }^{3}$ and Maurizio Brocchini ${ }^{1}$

\author{
AFFILIATIONS \\ ${ }^{1}$ Department I.C.E.A., Università Politecnica delle Marche, via Brecce Bianche 12, 60131 Ancona, Italy \\ ${ }^{2}$ Department of Mathematics, University of Oslo, P.O. Box 1053, Blindern, NO-0316 Oslo, Norway \\ ${ }^{3}$ DNV GL Oil \& Gas, P.O. Box 300, NO-1322 Høvik, Norway
}

a) Author to whom correspondence should be addressed: atlej@math.uio.no

\begin{abstract}
Experimental results of wave-induced vortex generation around a slender, vertical cylinder are discussed. Coherent vortices appear in long waves ( $k R \sim 0.1$, where $k$ is the wave number and $R$ is the cylinder radius) during the timing of the secondary load cycle, a strongly nonlinear component of the wave force acting on the cylinder, which is also measured. However, the secondary load cycle is also present in moderately long wave cases $(k R \sim 0.3)$ where there is no vortex formation. The measurement of vortex generation is enabled by particle image velocimetry. The flow downstream the cylinder, in three horizontal planes at different depths, is measured. The vortex formation that occurs in the long waves is attached to the cylinder in the form of thin vortex tubes. These appear symmetrically at angles of $40^{\circ}-45^{\circ}$ off the wave propagation direction. In one weak long wave case, several very thin vortex tubes appear along the back side of the cylinder. Vortex diameters are $20 \%$ of the cylinder diameter in four cases and 50\% of the cylinder diameter in one case. The measured vorticity emanates from the cylinder's boundary layer and is an order of magnitude stronger than the vorticity caused by wave breaking. Wave breaking reduces the vortex strength. The fact that the secondary load cycle appears without and with flow separation effects indicates that the load cycle is a gravity wave phenomenon that scales with the Froude number but that flow separation effects also contribute to the magnitude of such suction forces.
\end{abstract}

Published under license by AIP Publishing. https://doi.org/10.1063/1.5141131

\section{INTRODUCTION}

High-order nonlinear wave forces on vertical bottom fixed columns, typically for supporting offshore platforms and wind turbines, and wave-induced high-frequency structural vibrations have been actively studied for a few decades (Grue et al., 1993; 1994; Jefferys and Rainey, 1994; Faltinsen et al., 1995; Malenica and Molin, 1995; and Chaplin et al., 1997). Currently, the topics are highly relevant, not the least because of the new developments of offshore wind farms (Paulsen et al., 2014; Kristiansen and Faltinsen, 2017; Chen et al., 2018; and Riise et al., 2018a). Higher harmonic components of the wave force may induce resonant response, with structural vibrations well above the governing wave frequency.

The secondary load cycle (SLC), occurring at a frequency above the third harmonic, is among the high-order nonlinear wave forces (Riise et al., 2018b). The SLC consists of a peak in the load time series following the main cycle at a time corresponding to the minimum loading, when the wave crest leaves the column. These phenomena were first measured by Grue et al. (1993) and further investigated by Grue and Huseby (2002).

Currently, the role of possible vortex generation (VG) effects on the force and resonant responses due to steep wave events is debated. This was recently the object of computational investigations by Paulsen et al. (2014) and Kristiansen and Faltinsen (2017). Using an Open Foam adaptation, the high-order force simulations in steep regular waves at a finite water depth by Paulsen et al. (2014) showed that a highly nonlinear SLC occurs in relation to a downstream vortex formation. However, a slip boundary condition was used. Clearly, the vortex formation was not connected to the boundary layer dynamics. Later, Kristiansen and Faltinsen (2017) (Fig. 20), simulated a similar vortex formation under intermediate water depth conditions. These findings suggest that vortex cores have a diameter at least comparable to the cylinder radius. Differently, boundary layer separation effects were the driving mechanism in the simulations of Kristiansen and Faltinsen (2017). They 
used LES simulations resolving the boundary layer. Contrary to evidences from these studies, no flow separation effects were observed from the images of the water surface in the experiments on the SLC by Grue et al. $(1993 ; 1994)$ although they did not measure the vorticity.

In this paper, the vortex generation downstream of a slender cylinder exposed to steep wave events is measured together with the force. By arranging an experimental setup that combines particle image velocimetry (PIV) measurements over horizontal planes at different elevations below the free surface with the measurements of wave-induced force, we obtain information on (a) the vortex generation (VG) at the back side of the cylinder and (b) the occurrence of the secondary load cycle (SLC). The overview of the instantaneous vorticity fields and wave-exciting moment, derived from the post-processing of the laboratory measurements, is at the basis of an interpretation of the relationship between VG and the SLC.

This paper is organized as follows. Section II describes the laboratory experiments; in Sec. III, the main results and a discussion on them are presented; finally, in Sec. IV, some conclusions are provided.

\section{LABORATORY EXPERIMENTS}

The experiments were performed in the Hydrodynamics Laboratory at the University of Oslo. The wave flume, having a length of $25 \mathrm{~m}$ and a width of $0.5 \mathrm{~m}$, characterized by glassed sidewalls and bottom, is well suited for optical measurements. At one end of the tank, a hydraulic piston-type wavemaker has movements controlled by using a computer with preset voltage time series based on linear wavemaker theory. At the opposite end, a passive absorbing beach damps the wave reflection down to $3 \%$ of the amplitude of the incoming wave (Brown and Jensen, 2001 and Grue et al., 2003). However, the measurements of the tested waves impinging on the model were completed in a time window chosen so that they are not affected by any reflected waves from the beach.

\section{A. Physical model and instrumentation}

A cylinder model was installed on the centerline of the tank, at a distance of $10.9 \mathrm{~m}$ from the wavemaker, in order to preserve undisturbed flow conditions in the measuring area. It consisted in a transparent vertical surface-piercing cylinder of acryl with a diameter $\mathrm{D}=6 \mathrm{~cm}$, pivoted at the bottom by using a bilateral hinge; see Fig. 1(a). On the top, at $z_{a}=0.90$ above the bottom hinge, the cylinder was supported by a set of load cells (HBM Z6C2 with $10 \mathrm{~kg} \simeq 2$ $\mathrm{mV} \mathrm{V}^{-1}$, sampling rate $400 \mathrm{~Hz}$ ), indicated by FX1 and FX2 in this figure. From the load cells, the wave-exciting moment, with respect to the bottom hinge, was obtained. For further description and details about the cylinder setup, see the work of Riise et al. (2018a; 2018b). The time series of the wave elevation upstream the cylinder, without and with the cylinder present, were recorded by using a total of four acoustic wave gauges (UltraLab ULS Advanced Ultrasound, USS02/HFP, sampling rate $250 \mathrm{~Hz}$ ). In the wave elevation measurements, data dropouts occurred for the four steepest waves. In particular, the wave impact on the cylinder was captured by using the wave gauge WG3 located at the centerline, $6 \mathrm{~cm}$ upstream of the cylinder, and by using WG4 located $11 \mathrm{~cm}$ to the side of the cylinder. Two other wave gauges were also installed: WG1 and WG2 at a distance of $9 \mathrm{~m}$ and $15 \mathrm{~m}$ from the wavemaker, respectively. WG1 measured the incoming waves, and WG2 measured the transmitted waves (the results are not shown). The high accuracy of the instrumentation was recognized, as also underlined by Riise et al. (2018a), reporting an error lower than $0.01 \mathrm{Nm}$ for the load cells, corresponding to less than $3 \%$ with regard to the standard deviation
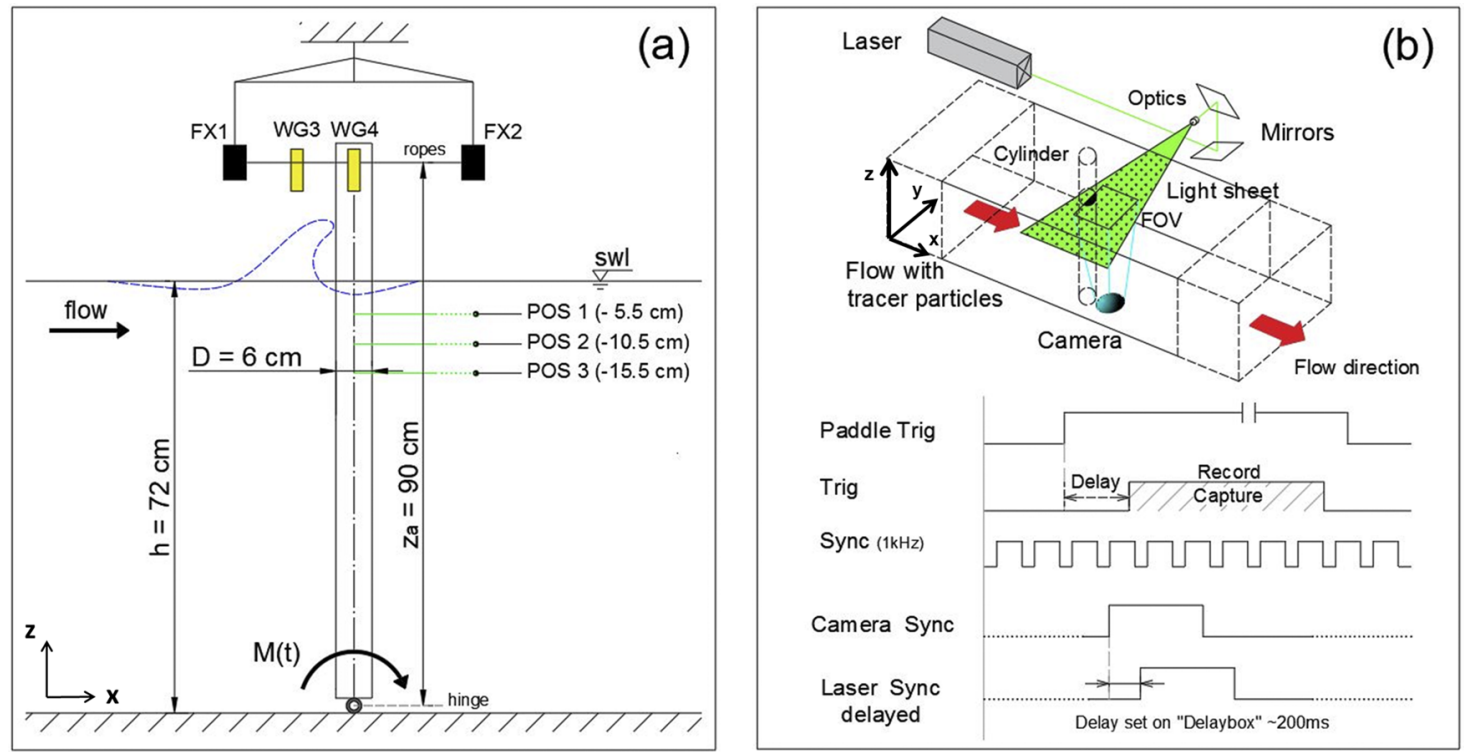

FIG. 1. Experimental setup. (a) Longitudinal view of the physical model (dimensions expressed in $\mathrm{m}$ ) and (b) PIV system arrangement with the timing diagram. 
of the measured load time series, and lower than $1 \mathrm{~mm}$ for the wave probes, i.e., less than $2 \%$ of the cylinder diameter.

Horizontal velocity vector components of the flow were measured behind the cylinder by employing a Particle Image Velocimetry (PIV) system, consisting of a camera, a laser beam as the illumination source, and a synchronizer controlled and monitored by using a computer, as illustrated in Fig. 1(b). A longitudinal beam originated by a Nd:YLF pulsed laser (Quantronix Darwin Duo $15 \mathrm{~mJ}$ at $3000 \mathrm{~Hz}$ ) placed laterally to the tank, through a suitable arrangement of mirrors and optics, was first deviated and then spread out into a thin and homogeneous horizontal laser sheet illuminating a $10 \times 10 \mathrm{~cm}^{2}$ field of view (FOV) to be observed. Three vertical positions at the back side of the cylinder, indicated by POS1, POS2, and POS3 in Fig. 1(a), were investigated, respectively, at $-5.5 \mathrm{~cm}$, $-10.5 \mathrm{~cm}$, and $-15.5 \mathrm{~cm}$ from the free surface, by shifting down the laser sheet $5 \mathrm{~cm}$ each time. The water was seeded by neutrally buoyant polyamide particles (the same density as water) with a diameter of $50 \mu \mathrm{m}$. A high-speed CMOS camera (Photron FASTCAM SA5 $1000 \mathrm{~K}-\mathrm{M} 2$ ) of $1024 \times 1024$ resolution, located below the wave tank, facing upwards and normal to the laser sheets, captured images of the seeded FOV at a sampling rate of $1000 \mathrm{~Hz}$. The measurements of wave elevation, wave-exciting moment, and the velocity fields were acquired through a period of $10 \mathrm{~s}$ that covered the wave impact for each test. Timing and synchronization of all among the instruments were carried out using the paddle trigger signal as an external input signal and varying the pre-established starting delay with relation to the wave period. Details of the functioning of the synchronization system are illustrated by the timing diagram in Fig. 1(b). Note that a delay of $200 \mathrm{~ms}$ between the synchronization pulse of the camera and that of the laser was set in order to ensure that the laser light pulses occurred within the exposure time of the camera.

\section{B. Wave generation and wave characteristics}

Steep wave events at the cylinder position were generated employing a wave frequency-focusing technique, which is recognized as a suitable method to simulate the natural mechanism of generation of breaking waves in deep waters (Chaplin et al., 1997). This technique, based on the linear dispersion theory, leads to designing a wave packet that converges up to the desired time and spatial location, generating a single large wave. The frequencyfocusing technique was already implemented in the tank by Brown and Jensen (2001), and its high accuracy has been proven by previous studies (Huseby and Grue, 2000; Jensen et al., 2001; and Jensen and Grue, 2002). Seven different waves were tested (Table I), detecting four different scenarios of impact: (i) impact of strong spilling breakers, (ii) impact of weak spilling breakers, (iii) impact of plunging breakers, and (iv) impact of non-breaking waves. Each of the wave events was defined by the crest height $\eta_{c}$ and the trough-totrough period $\mathrm{T}_{t t}$. Three repetitions of the surface elevation timehistories at WG3 with no cylinder were performed, obtaining the ensemble mean of $\eta_{c}$ and $\mathrm{T}_{t t}$. An additional measure of the wave period was obtained from the time series of the overturning moment acting on the cylinder. The zero up-crossing period of this moment, $T_{f}$, is actually very close to $T_{t t}$. The average of these two periods is used, i.e., $(1 / 2)\left(T_{t t}+T_{f}\right)$. In dimensionless terms, this period becomes $T_{a v}^{*}=(1 / 2)\left(T_{t t}+T_{f}\right) \sqrt{g / D}$, where for the long wave cases $\mathrm{A} 1-\mathrm{A} 5$, the period is in the range $T_{a v}^{*} \sim 13-16$ and for the shorter wave cases B1-B2, the period is $T_{a v}^{*} \sim 8$ (Table I). The wave number $k$ and the first-order wave slope $\epsilon$ were defined in each of the wave events from (Grue et al., 2003)

$$
\omega^{2} /(g k)=1+\epsilon^{2}, \quad k \eta_{c}=\epsilon+\frac{1}{2} \epsilon^{2}+\frac{1}{2} \epsilon^{3}
$$

\{where $\omega=2 \pi /\left[(1 / 2)\left(T_{t t}+T_{f}\right)\right]$ and $g$ is the acceleration due to gravity\}, resulting from the third order Stokes wave theory used as a reference. A constant water depth of $h=0.72 \mathrm{~m}$ was used in all the tests, giving $k h>1.6$. In particular, $2<k h<7$ represents deep water waves, while $k h=1.8$ in case Al represents an intermediate water wave. From them, the dimensionless wave slope $k \eta_{c}$ and the dimensionless wave number with respect to the cylinder radius $k R$ were derived. Likewise, the horizontal fluid velocity below crest, estimated by $U_{c}=\epsilon \sqrt{g / D} \exp \left(k \eta_{c}\right)$, obtains the Froude number $F r=U_{c} / \sqrt{g D}$, Keulegan-Carpenter (KC) number $K C=U_{c}\left[(1 / 2)\left(T_{t t}\right.\right.$ $+T_{f}$ ) $] / D$, and Reynolds number $R e=U_{c} D / v$ (with $v$ being the fluid kinematic viscosity), where, in the present experiments, $0.13<k \eta_{c}$ $<0.45,0.074<k R<0.29,0.37<F r<1.05,5.3<K C<13.8$, and $17000<\operatorname{Re}<46000$.

TABLE I. Organized parameters of the tested waves in light of the results available: $\eta_{c}-$ surface elevation at the crest measured by using WG3, $T_{t t}$-trough-to-trough period, $T_{f}$-zero up-crossing period of the force history, $T_{a v}^{*}-$ dimensionless average period, $k \eta_{c}$-wave slope, $k R$-wave number, $K C$-Keulegan-Carpenter number, and Fr-Froude number. VG means vortex generation, while SLC means the secondary load cycle, whose presence is indicated by " $Y$ " (yes) or "N" (no).

\begin{tabular}{llcccccccccc}
\hline \hline Cases & \multicolumn{1}{c}{ Description } & $\eta_{c}(\mathrm{~cm})$ & $T_{t t}(\mathrm{~s})$ & $T_{f}(\mathrm{~s})$ & $T_{a v}^{*}$ & $k \eta_{c}$ & $k R$ & \multirow{2}{*}{ KC } & Fr & VG & SLC \\
\hline A1 & Mild steep non-br. & 5.39 & 1.24 & 1.28 & 16.3 & 0.13 & 0.074 & 6.0 & 0.37 & $\mathrm{Y}$ & $\mathrm{N}$ \\
$\mathrm{A} 2$ & Strong spilling br. & 7.63 & 1.07 & 1.08 & 13.8 & 0.25 & 0.10 & 8.8 & 0.64 & $\mathrm{Y}$ & $\mathrm{Y}$ \\
$\mathrm{A} 3$ & Strong spilling br. & 7.98 & 0.99 & 1.02 & 12.9 & 0.30 & 0.11 & 9.4 & 0.73 & $\mathrm{Y}$ & $\mathrm{Y}$ \\
$\mathrm{A} 4$ & Steep non-br. & 9.21 & 1.12 & 1.20 & 14.9 & 0.26 & 0.085 & 10.7 & 0.72 & $\mathrm{Y}$ & $\mathrm{Y}$ \\
$\mathrm{A} 5$ & Plunging br. & 11.3 & 0.98 & 1.07 & 13.1 & 0.39 & 0.10 & 13.8 & 1.05 & $\mathrm{Y}$ & $\mathrm{Y}$ \\
$\mathrm{B} 1$ & Weak spilling br. & 4.30 & 0.61 & 0.63 & 7.8 & 0.41 & 0.29 & 5.3 & 0.67 & $\mathrm{~N}$ & $\mathrm{Y}$ \\
$\mathrm{B} 2$ & Weak spilling br. & 4.70 & 0.59 & 0.63 & 7.9 & 0.45 & 0.29 & 5.7 & 0.75 & $\mathrm{~N}$ & $\mathrm{Y}$ \\
\hline \hline
\end{tabular}




\section{Data analysis}

Wave surface elevation profiles with data dropouts (at the locations where the wave reached such large values of steepness that could not be detected by using the acoustic gauges) were reconstructed by using a curve fitting function that provided the piecewise polynomial form of a shape-preserving piecewise cubic Hermite interpolant. A low pass filter was used on the force data, with a cutoff frequency of $20 \mathrm{~Hz}$, considered to be well above the significant wave and load frequencies. The PIV analysis has been performed for each pair of consecutive frames, previously masked from the visible cylinder portion and from the saturated parts. An automatic resizing of the interrogation subwindow, initially pre-set at $32 \times 32$ pixels with an overlap of $50 \%$, was adopted. The search range was set to go from -16 to 16 pixels. The cross-correlation function was used to find the optimal match of the displacements between particles. Noise filters and the detection/replacement of outlier vectors were applied.

\section{RESULTS AND DISCUSSION}

Three realizations of each test were carried out for all the wave cases listed in Table I, resulting in a total of 63 tests performed. For each wave, we considered the incoming wave, more precisely the surface elevation at the cylinder provided by WG4, and the waveexciting moment, both obtained as the mean over the three repetitions of the test. Furthermore, the vorticity maps derived from PIV, superposed to the corresponding velocity fields, were studied. Dimensionless parameters have been adopted for the representation of the results. The dimensions of the FOV and the surface elevation $\eta$ are normalized by $D$, the wave-exciting moment $M$ is normalized by $\rho g z_{a} D^{3}$ (with $\rho$ as the density of water), and the time $t$ is normalized by $1 / \sqrt{g / D}$.

\section{A. Secondary load cycle}

The occurrence of the secondary load cycle (SLC) phenomena was recognized, more or less clearly, from the wave-exciting moment series for all the wave cases, except for case A1 (Table I). In detail, from the high harmonic force analysis at frequency $\omega$ exceeding 3.5 times the fundamental frequency, only a very weak SLC occurred for case A1. By using the SLC ratio, defined as the ratio between its magnitude over the peak-to-peak force (Grue, 2002), the intensity of the observed SLC phenomena was estimated between 3\% and 6\%. No apparent distinction in SLC occurrence was seen in the long and moderately long wave regimes. Similarly, in both A2-A5 and B1-B2, respectively, the SLC occurred at about one quarter wave period later than the main peak of the moment, lasting for about $15 \%$ of the wave period, in agreement with the studies of Grue et al. (1993) and Grue and Huseby (2002). Furthermore, the SLC was found for Fr $>0.6$, $k \eta_{c}>0.25$, and $k R>0.08$ in the present experiments. This is within the range of values for the SLC occurrence reported in the literature (Chaplin et al., 1997; Grue and Huseby, 2002; and Riise et al., 2018b and 2018a).

\section{B. Vortex generation}

By physical laboratory experiments, our main purpose is to document the appearance of possible flow separation and vortex formation for a flow still largely debated in the science community. Firm documentation of the processes is lacking. The flow separation and vortex formation are induced by long waves compared to the cylinder radius $(k R \sim 0.1)$ that are moderately strong to strong $\left(k \eta_{c}\right.$ $\sim 0.13-0.45)$. The shorter wave cases $(k R \sim 0.3)$ did not exhibit vortex formation.

The PIV measurements, obtained in three horizontal planes at depths $5.5 \mathrm{~cm}, 10.5, \mathrm{~cm}$ and $15.5 \mathrm{~cm}$ below the mean water surface, enable the visualization of the degree of two-dimensionality of the vorticity.

Present measurements of vorticity are compared to the available numerical calculations. The wave-cylinder parameters in the present case A1 are close to the case studied by Paulsen et al. (2014). Cases A2 and A3 are close to a flow separation example included in Sec. 4.3.1 of the work of Kristiansen and Faltinsen (2017). Wave breaking may induce three-dimensional, vertical circulation cells [e.g., in the work of Kirby and Derakhtib (2019)] and is discussed. Cases B1 and B2 are compared to the experiments where the secondary load cycle-termed a secondary oscillation in the force-recordings-was first measured (Grue et al., 1993; 1994).

An estimate of the vorticity is obtained by evaluating circulation integrals in each horizontal plane, $\oint(u, v, w) \cdot d \mathbf{s}$. The $d \mathbf{s}$ is taken as the vectorial grid spacing of the PIV analysis in each of the horizontal planes. The vorticity $\omega$ is obtained by dividing the area enclosed by the integration contour.

Vortex formation behind the cylinder is illustrated at four specific time instants, where time I corresponds to the main peak of the wave-exciting moment or maximum input wave elevation at the cylinder position. The vortex formation may be connected to the secondary load cycle (SLC). Time II is thus at the start of the SLC, time III corresponds to the peak of the SLC, and time IV is at the end time of the SLC.

In case A1, Fig. 2, the incoming wave is a long, non-breaking wave with $k R=0.074, T_{a v}^{*}=16.3$, and $k \eta_{c}=0.13$. There is no or only a weak SLC. At wave maximum, where also the horizontal velocity is at maximum, the experiment shows clear formation of vorticity in the thin boundary layer of the cylinder. The vorticity is symmetrical about the vertical centerplane of the wave tank. The vorticity, observed similarly at all vertical positions POS1, POS2, POS3, is negative on the left, upper back side of the cylinder and positive on the right, lower side. The magnitude is up to $200 \mathrm{~s}^{-1}$.

Two main vertical vortex tubes and an additional small one, all with negative orientation, are formed at the upper back side of the cylinder. Two tubes plus one small vortex tube, of positive orientation, are correspondingly formed at the lower side of the cylinder, at time instants II $^{\prime}$ and III'. The vortex diameter is up to $0.2 \mathrm{D}$. The well organized vortex tubes break down at time $\mathrm{IV}^{\prime}$.

Kristiansen and Faltinsen (2017) (Sec. 4.3.1) proposed similar wave/cylinder parameters as our case A1, with $k R=0.13, T_{a v}^{*}=12.5$, and $k \eta_{c}=0.13$. Their wave is somewhat shorter. In order to investigate the effect on the free surface due to flow separation, they proposed a simplified method. The flow separation effect itself was obtained by two-dimensional LES-simulations with a no-slip condition at the cylinder, without any wave. Their counter-rotating vortex had a diameter $0.5 D$. Their vortex contributed to a load history containing several harmonics, different from our experiments. Present measurements, with several thin vortex tubes and no measured 


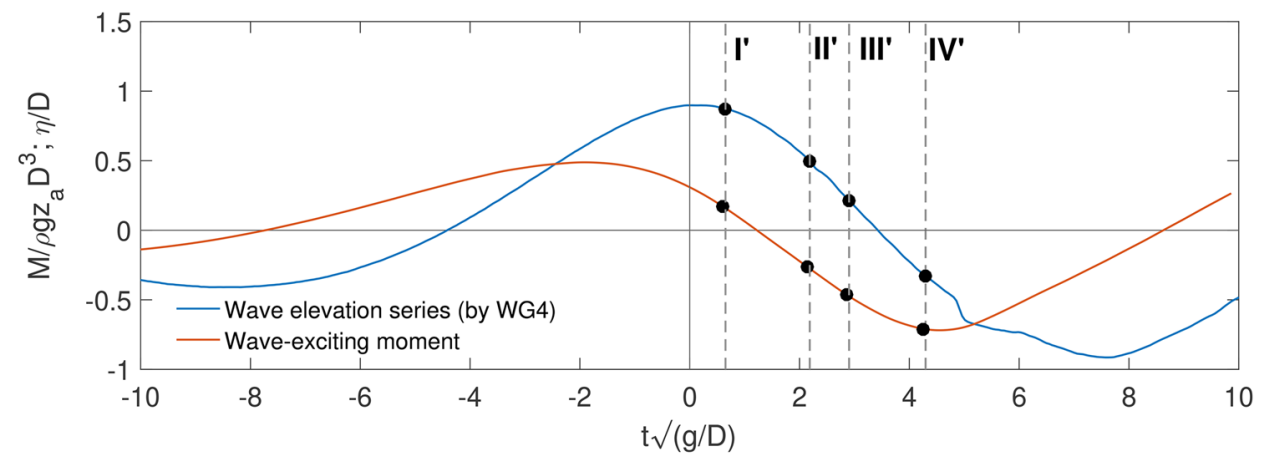

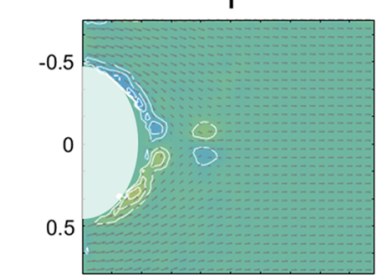
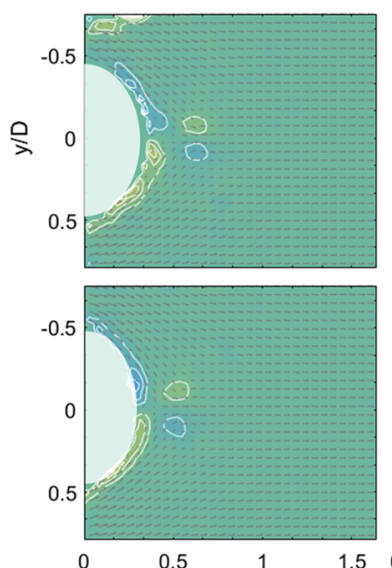

(a)

II'
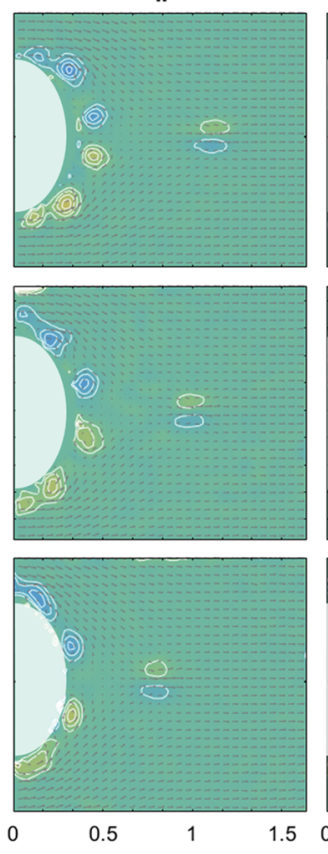

III
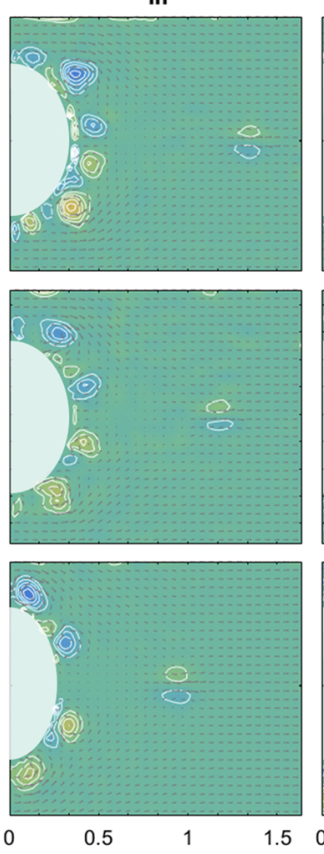

IV'

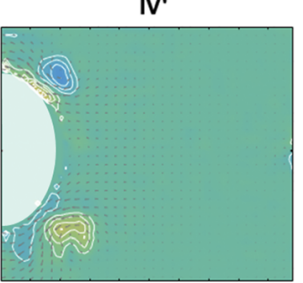

[1/s]
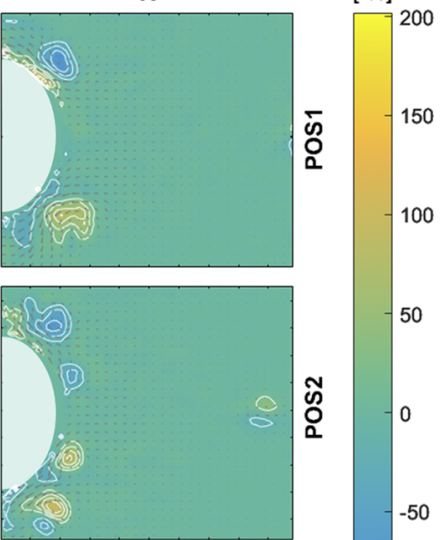

ฒั
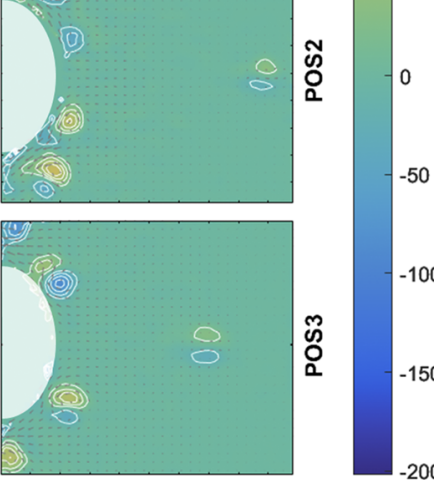

\%

(b)

FIG. 2. Vortex evolution for case A1. (a) Time histories of wave elevation (in blue) and the wave-exciting moment (in red). (b) Evolution of instantaneous vorticity at times $I^{\prime}-I V^{\prime}$ and at horizontal planes POS1-POS3. Incident flow is from left to right.

secondary load cycle, differ much from the assumed vortex mimicked by Kristiansen and Faltinsen (2017).

Present cases A2 and A3 have very similar wave parameters, where results for case $\mathrm{A} 3$ are discussed; see Fig. 3. The input spilling wave has parameters $k R=0.11, T_{a v}^{*}=12.9$, and $k \eta_{c}=0.30$. The force history displays a SLC (see II to III). The main features of the vorticity are the formation of one vortex of negative rotation at the upper back of the cylinder and an image vortex at the lower back, of positive rotation, at times II and III. The vorticity strength is $\pm 100 \mathrm{~s}^{-1}$, approximately half of case A1. Vortices of the diameter of $0.2 D$ are located at $\pm 45^{\circ}$, approximately. The vortices are similar at the planes 1 and 2 (at $-5.5 \mathrm{~cm}$ and $10.5 \mathrm{~cm}$ below the mean water line), where obviously the vortex tubes are somewhat tilted. The vortices partially breakup at time IV.

In Fig. 15 of the work of Paulsen et al. (2014), their wave parameters correspond to $k R=0.1, T_{a v}^{*}=16.8$, and $k \eta_{c}=0.3$ using the present notation and are close to those of our cases A2 and A3. While the vorticity in our case is driven by the viscous effects in the cylinder's boundary layer, their inviscid simulations obtained vorticity by wave breaking. Their calculated vorticity, of magnitude up to $5 \mathrm{~s}^{-1}$, exhibited a vortex cell of diameter $0.4 D$ next to the centerplane of the same orientation as our vortex. This vorticity strength is typical for vorticity driven by wave breaking, where, e.g., Kirby and Derakhtib (2019), Fig. 11, obtained a vorticity in the range $\pm 3 \mathrm{~s}^{-1}$ under 


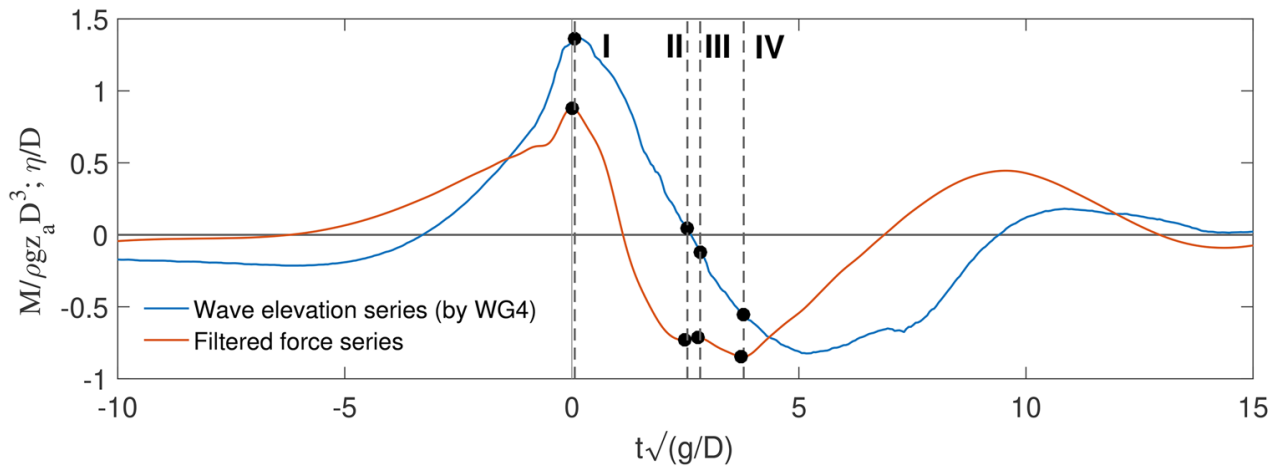

(a)
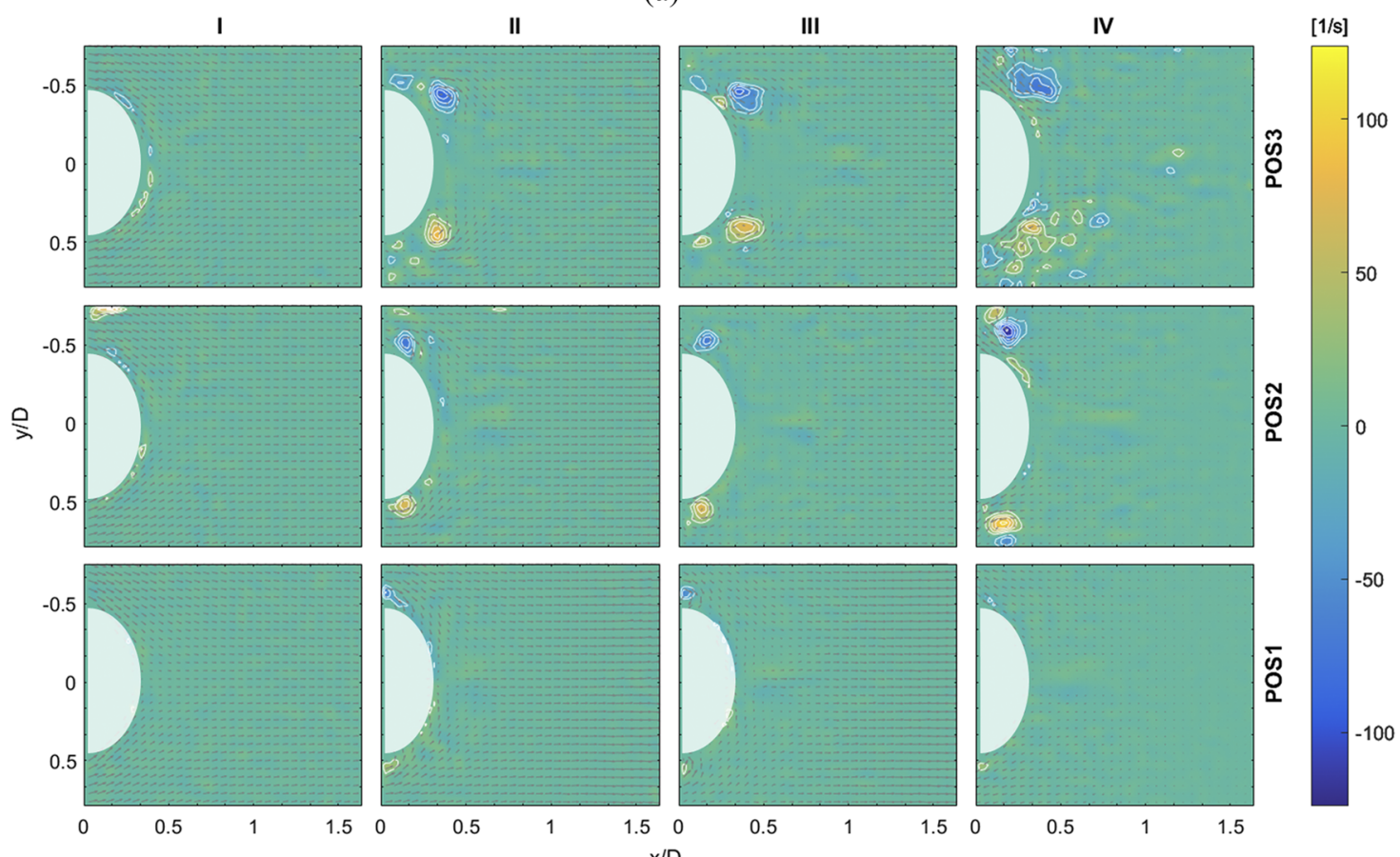

(b)

FIG. 3. SLC dynamics for case A3. (a) Time histories of wave elevation (in blue) and the wave-exciting moment (in red). (b) Evolution of instantaneous vorticity at times I-IV characteristic of SLC dynamics and at horizontal planes POS1-POS3. Incident is flow from left to right.

laboratory conditions. This means that the wave breaking driven vorticity is just a fraction of the vorticity that emanates from the boundary layer of the cylinder. Note that the vorticity scales according to the inverse of the wave period. This is of the order of $1 \mathrm{~s}$ in the laboratory and of $10 \mathrm{~s}$ in the field.

The input wave in case A4 is long, strong, and non-breaking (Fig. 4). The parameters are $k R=0.085, T_{a v}^{*}=14.9$, and $k \eta_{c}=0.26$. The force history has a SLC. The vortex cells, with a diameter up to $0.5 \mathrm{D}$, are located at approximately $40^{\circ}-45^{\circ}$ and are wider than those in case A3. The vorticity is relatively similar in all planes 1-3. The vorticity is up to $\pm 150 \mathrm{~s}^{-1}$. The vorticity structure is completely breaking down at time IV.
In case A5, Fig. 5, the input wave is a plunging breaker. Parameters are $k R=0.10, T_{a v}^{*}=13.1$, and $k \eta_{c}=0.39$. This case exhibits a vortex formation similar to that in case A3 (and A2). The vorticity is up to $\pm 100 \mathrm{~s}^{-1}$, and vortex diameter is up to $0.2 D$. Location is at $40^{\circ}-45^{\circ}$. Vortex tubes collapse at time instant IV.

In the relatively shorter wave cases B1 and B2, where the input waves were weakly spilling and wave parameters are $k R=0.29$, $T_{a v}^{*} \simeq 8$, and $k \eta_{c}=0.43 \pm 0.02$, we did not measure flow separation at the cylinder. A SLC in the force history was measured, however. Note that the wavelength and period in cases B1 and B2 are close to those in the work of Grue et al. $(1993 ; 1994)$, where $k R=0.24$ and $T_{a v}^{*} \simeq 9.5$, where a secondary oscillation in the force history, later 


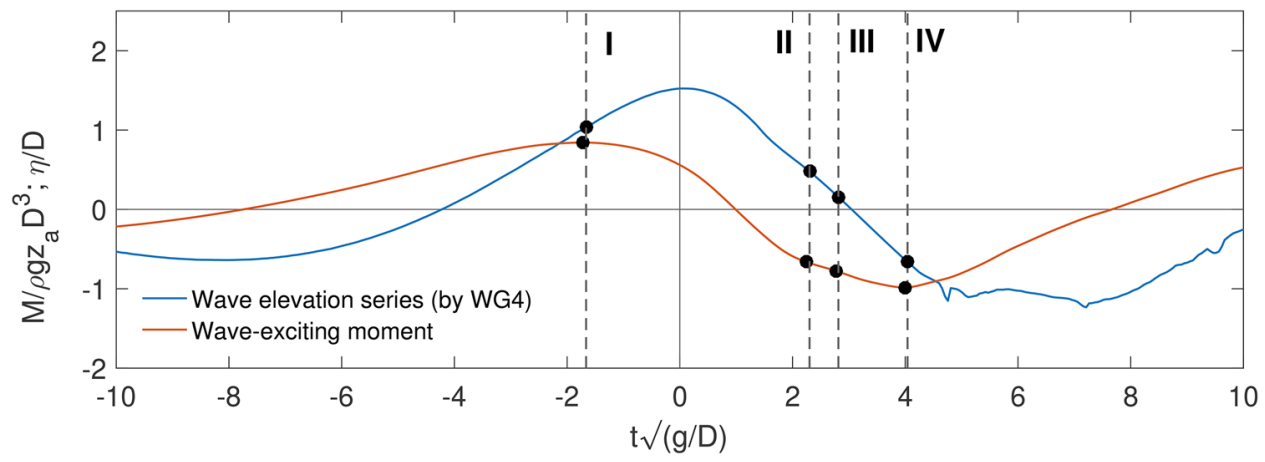

(a)

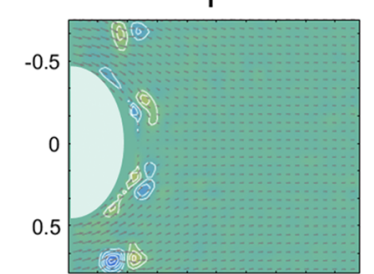

III
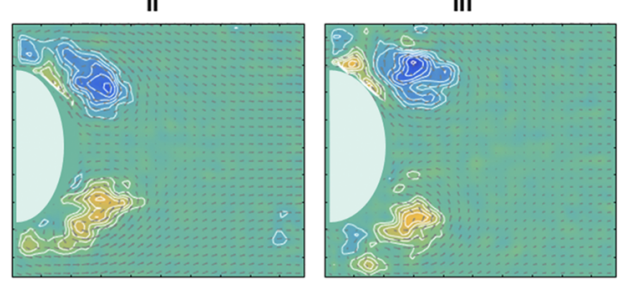

IV

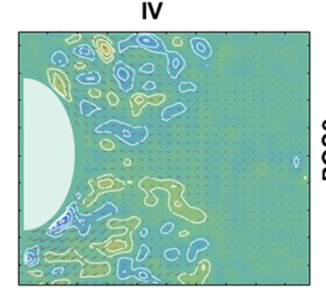

$[1 / \mathrm{s}]$
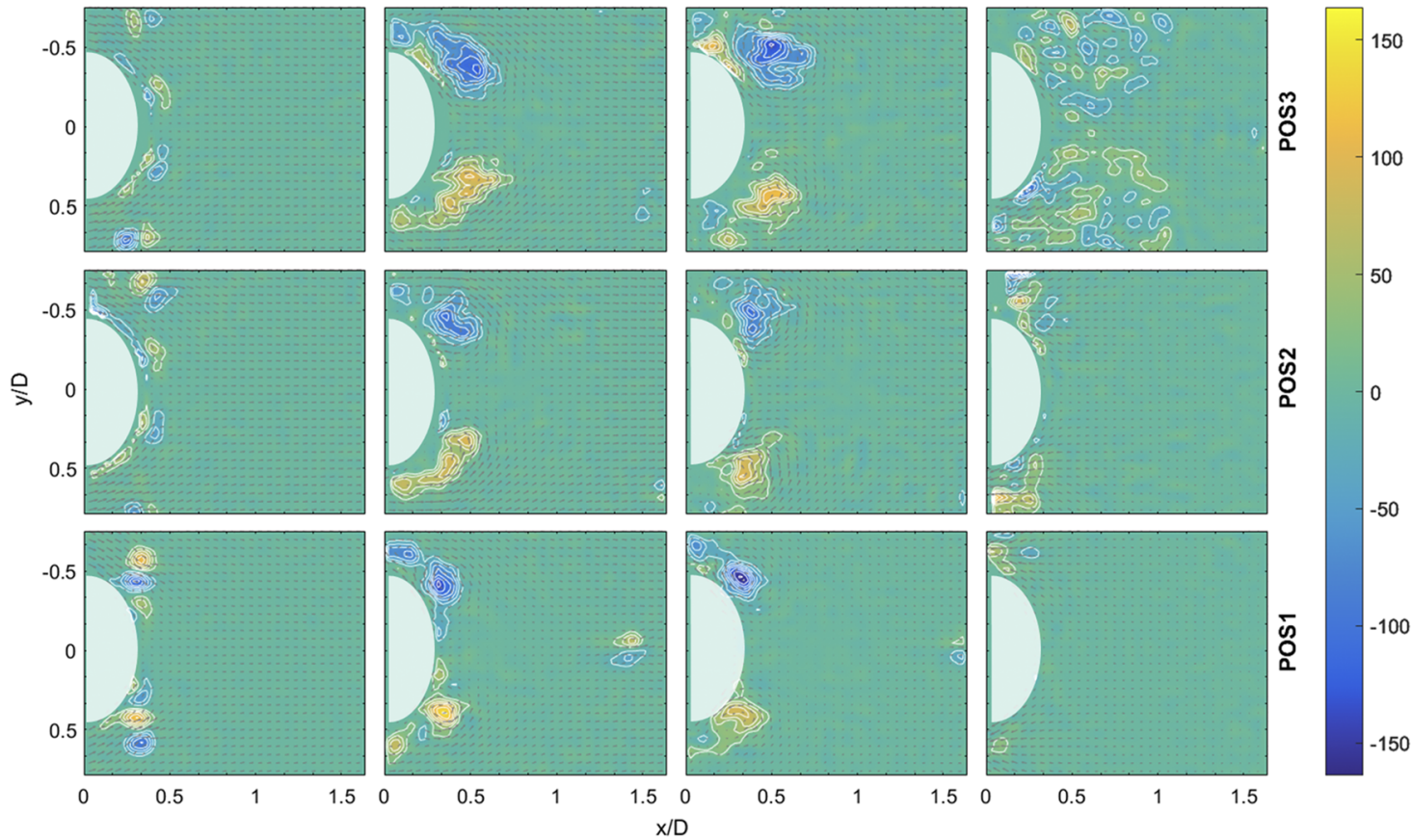

(b)

FIG. 4. Vortex evolution for case A4. (a) Time histories of wave elevation (in blue) and the wave-exciting moment (in red). (b) Evolution of vorticity at times I'-IV' and at horizontal planes POS1-POS3. Incident is flow from left to right.

denoted by the SLC, was first measured and discussed. In that investigation, the SLC appeared for $k \eta_{c}>0.28$. A corresponding Froude number was $\mathrm{Fr}>0.4$. Grue et al. $(1993 ; 1994)$ suggested that the secondary force oscillation was due to a suction force. They did not observe any flow separation in the experiments. This is confirmed by the present measurements.

Regarding the three-dimensionality of the wave-cylinder induced vorticity, we note that the measured vorticity appears in a symmetrical manner with respect to the vertical centerplane of the wave tank. This means that the velocity circulation along a closed curve in a vertical plane, orthogonal to the wave propagation direction, where also the integration curve is symmetrical with respect to the centerplane, is zero because of symmetry. The measurement of the vertical velocities and the horizontal vorticity is left for future studies.

The main results from the seven cases are that vortices appear symmetrically with respect to the vertical centerplane of the wave tank.

In the longer waves, with $k R \sim 0.074-0.11$ and $T_{a v}^{*} \sim 13-16$, the force history exhibits a SLC, whereas in the weaker case A1, this is absent. In these waves, and during the timing of the SLC, a pair of vortices appear at the upper and lower back of the cylinder. The vortices are generated in the cylinder's boundary layer. Vortex tubes are located at $40^{\circ}-45^{\circ}$ from the centerplane, during the timing of the SLC. The vorticity is the strongest in the nonbreaking cases where the magnitude is up to $200 \mathrm{~s}^{-1}$. Vortices break down when the SLC 


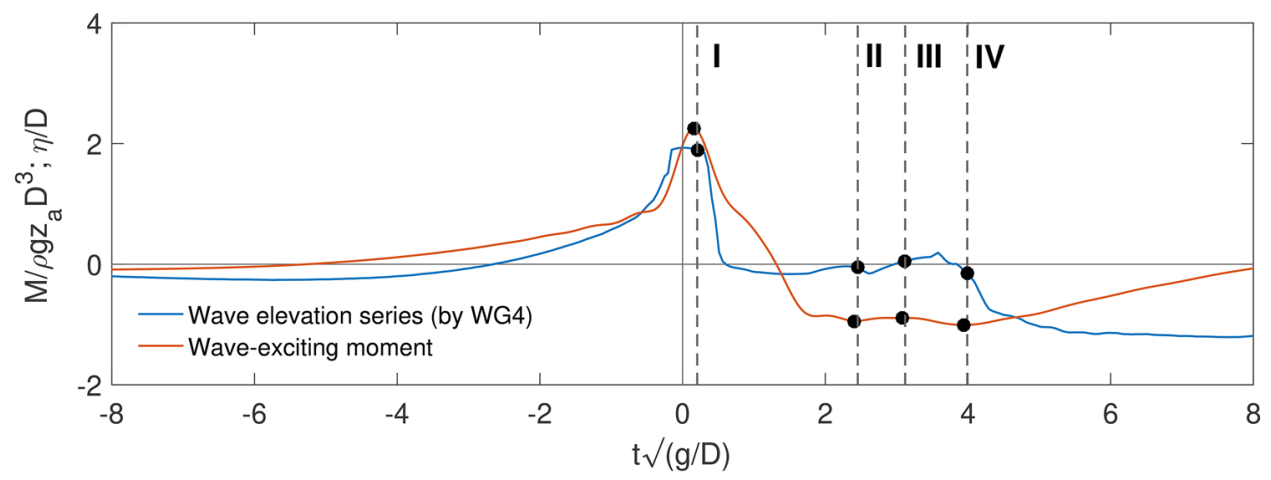

(a)
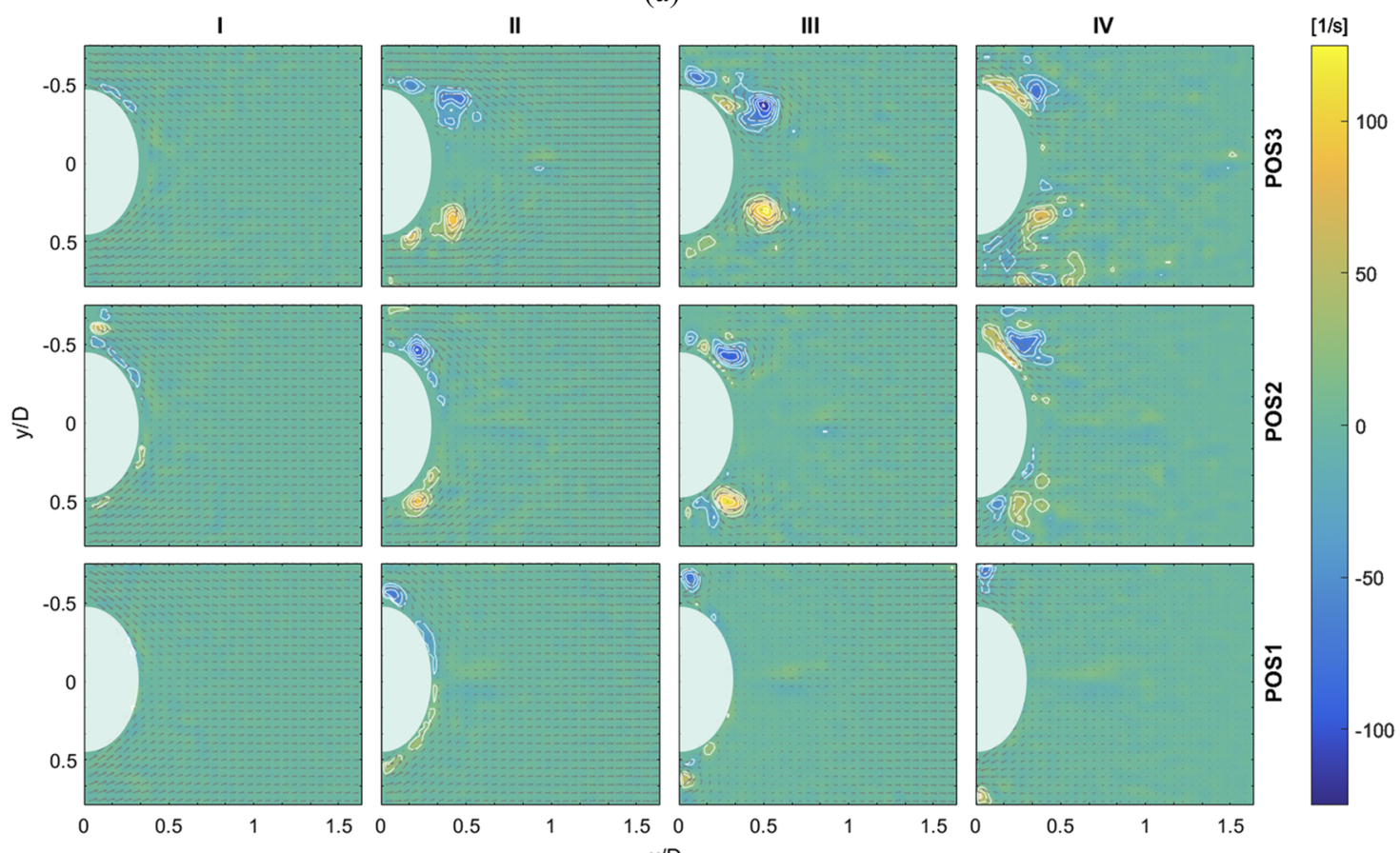

(b)

FIG. 5. SLC dynamics for case A5. (a) Time histories of wave elevation (in blue) and the wave-exciting moment (in red). (b) Evolution of instantaneous vorticity at times I-IV characteristic of the SLC dynamics and at horizontal planes POS1-POS3. Incident flow is from left to right.

is over. In the weak case A1, where the SLC is marginal, the vorticity appears in the form of several thin vortex tubes.

Breaking of the input wave reduces the vorticity driven by the cylinder's boundary layer. Vorticity driven by wave breaking is up to $5 \mathrm{~s}^{-1}$ and is just a fraction of the vorticity driven by the cylinder's boundary layer.

In the shorter wave cases $\mathrm{B} 1$ and $\mathrm{B} 2$, with $k R=0.29$ and $T_{a v}^{*} \sim 8$, there is a SLC; however, we have not been able to measure any vortex formation.

The vortex formation in the longer wave cases occurs during the timing of the SLC. The measured vorticity and vortex tubes obviously have an effect on the velocity field. This effect is inherent in the contribution to the pressure force on the cylinder. The vortex formation reduces the pressure behind the cylinder.

Formation of vorticity is not necessary for the presence of a SLC, whereas in the shorter wave cases, this appears in the force history, where no vortex formation is measured. The SLC is driven by gravity wave effects, both with or without vortex formation.

Vortex formation in wavy flow is commonly evaluated in terms of the Keulegan-Carpenter $(K C)$ number, where effects on the forces appear above a certain value. Relating to present experiments, a value of $K C=5$ is suggested. On the other hand, gravity wave effects obey a Froude number scaling, where $\mathrm{Fr}=0.4$ refers to a gravity wave effect at the scale of the cylinder diameter. 


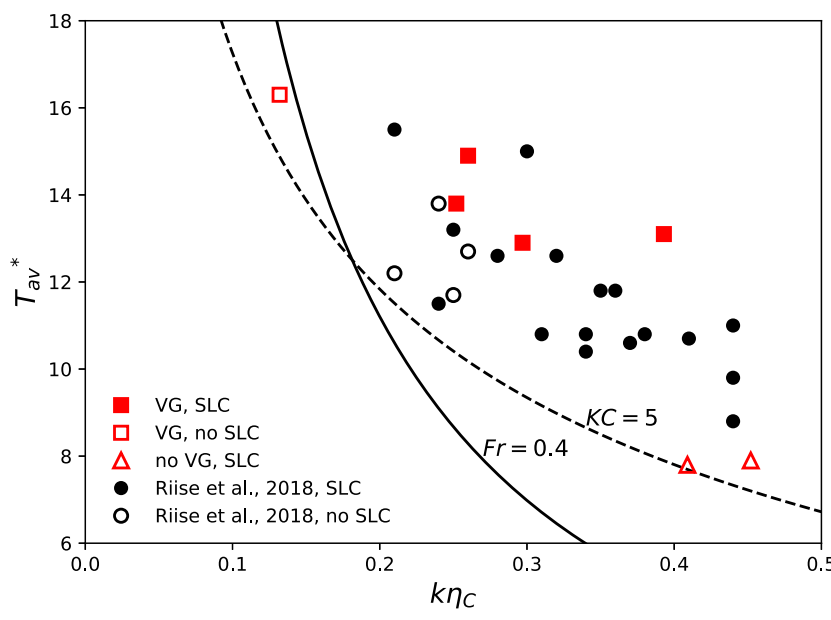

FIG. 6. Extreme response event identification. Experimental data (red markers) and data from the work of Riise et al. (2018a) (black dots). Open square: VG occurrence with no SLC (case A1), filled square: VG and SLC (cases A2-A5), open triangle: no vorticity and the presence of SLC (cases B1-B2), dashed line: $K C=0.5$, and continuous line: $F r=0.4$. Adapted from the work of Rilse et al., 2018b, Fig. 10(d).

Present observations of the SLC are plotted together with the results from the work of Riise et al. (2018b), Table 3, obtained in random waves (Fig. 6). All of the present cases occur for $K C>5$, where vortex generation was not measured in B1 and B2, however. Similarly, present experiments show that a SLC occurs for $F r>0.4$, indicating surface gravity wave effects at the scale of the cylinder diameter (Grue et al., 1993; 1994).

\section{CONCLUSIONS}

An experimental study with a bottom hinged cylinder exposed to long or moderately long waves that are moderately steep to steep and both non-breaking and breaking has been performed. The water depth is large or intermediate. The aim has been to study vortex generation (VG) and its possible connection with the secondary load cycle (SLC). Vorticity maps of the downstream flow by using the PIV technique have been obtained over three different horizontal planes (POS1-POS3). In moderately long waves $(k R \sim 0.3)$, no vortex formation was found during the presence of the SLC in the force history, which also was measured. However, in the long waves $(k R$ $\sim 0.1$ ), vortex formation has been found to take place. The vortex formation appears during the timing of the SLC. The vortex formation generally collapses at the end of this cycle. Vortices emanate from the cylinder's boundary layer in the form of vertical vortex tubes attached to the back side of the cylinder at $40^{\circ}-45^{\circ}$ from the wave propagation direction. The vortex diameter is $0.2 D$ in four among the five cases and $0.5 D$ in the strongest, non-breaking case. Wave breaking reduces the vorticity. In one long wave case of moderate amplitude, where the SLC is absent or very small, the vortex formation appears in the form of several thin vortex tubes along the cylinder contour, at the back side. In this case, the organization of vorticity lasts longer than the typical SLC period.
The measured vorticity due to the boundary layer effect of the cylinder is an order of magnitude larger than the vorticity generated by wave breaking. The vorticity measured herein appears fundamentally different from the computational results by Paulsen et al. (2014) and Kristiansen and Faltinsen (2017). The absence of vorticity in the moderately long wave case with $k R \sim 0.3, k \eta_{c} \sim 0.43$, during the presence of the SLC, concurs with the first descriptions by Grue et al. (1993; 1994), who did not observe flow separation during the SLC.

The vortex formation obviously contributes to the SLC on the cylinder exposed to the long waves. However, the SLC is also present in the shorter waves where no vortex formation takes place. This suggests that the SLC is driven mainly by gravity wave effects.

\section{ACKNOWLEDGMENTS}

The authors are deeply grateful to Head Engineer O. Gundersen for the technical assistance during the experiments. Professor A. Stocchino and G. Besio from Universitá degli Studi di Genova are thanked for the help with PIV data analysis.

\section{REFERENCES}

Brown, M. G. and Jensen, A., "Experiments on focusing unidirectional water waves," J. Geophys. Res.: Oceans 106(C8), 16917-16928, https://doi.org/ 10.1029/2000jc000584 (2001).

Chaplin, J. R., Rainey, R. C. T., and Yemm, R. W., "Ringing of a vertical cylinder in waves," J. Fluid Mech. 350, 119-157 (1997).

Chen, L. F., Zang, J., Taylor, P. H., Sun, L., Morgan, G. C. J., Grice, J., Orszaghova, J., and Tello Ruiz, M., "An experimental decomposition of nonlinear forces on a surface-piercing column: Stokes-type expansions of the force harmonics," J. Fluid Mech. 848, 42-77 (2018).

Faltinsen, O. M., Newman, J. N., and Vinje, T., "Nonlinear wave loads on a slender vertical cylinder," J. Fluid Mech. 289(1), 179 (1995).

Grue, J., "On four highly nonlinear phenomena in wave theory and marine hydrodynamics," Appl. Ocean Res. 24(5), 261-274 (2002).

Grue, J., Bjørshol, G., and Strand Ø., Higher Harmonic Wave Exciting Forces on a Vertical Cylinder (Matematisk Institutt, Universitetet i Oslo, 1993), Vol. in Preprint, pp. 1-30.

Grue, J., Bjørshol, G., and Strand, O., "Nonlinear wave loads which may generate 'ringing' responses of offshore structures," in Proceedings of the 9th International Workshop on Water Waves and Floating Bodies, 1994.

Grue, J., Clamond, D., Huseby, M., and Jensen, A., "Kinematics of extreme waves in deep water," Appl. Ocean Res. 25(6), 355-366 (2003).

Grue, J. and Huseby, M., "Higher-harmonic wave forces and ringing of vertical cylinders," Appl, Ocean Res. 24(4), 203-214 (2002).

Huseby, M. and Grue, J., “An experimental investigation of higher-harmonic wave forces on a vertical cylinder," J. Fluid Mech. 414, 75-103 (2000).

Jefferys, E. R. and Rainey, R. C. T., "Slender body models of TLP and GBS 'ringing,", in Seventh International Conference on the Behaviour of Offshore Structures (Pergamon, 1994), pp. 587-606.

Jensen, A. and Grue, J., "A note on the difference in the speed of gravity waves in a physical and numerical wave tank," Wave Motion 36(1), 41-48 (2002).

Jensen, A., Sveen, J. K., Grue, J., Richon, J.-B., and Gray, C., “Accelerations in water waves by extended particle image velocimetry," Exp. Fluids 30(5), 500-510 (2001).

Kirby, J. and Derakhti, M., “Short-crested wave breaking,” Eur. J. Mech.: B/Fluids 73, 100-111 (2019).

Kristiansen, T. and Faltinsen, O. M., "Higher harmonic wave loads on a vertical cylinder in finite water depth," J. Fluid Mech. 833, 773-805 (2017). 
Malenica, Š. and Molin, B., "Third-harmonic wave diffraction by a vertical cylinder," J. Fluid Mech. 302, 203-229 (1995).

Paulsen, B. T., Bredmose, H., Bingham, H. B., and Jacobsen, N. G., "Forcing of a bottom-mounted circular cylinder by steep regular water waves at finite depth,” J. Fluid Mech. 755, 1-34 (2014).
Riise, B. H., Grue, J., Jensen, A., and Johannessen, T. B., "A note on the secondary load cycle for a monopile in irregular deep water waves," J. Fluid Mech. 849, R1 (2018a).

Riise, B. H., Grue, J., Jensen, A., and Johannessen, T. B., "High frequency resonant response of a monopile in irregular deep water waves," J. Fluid Mech. 853, 564-586 (2018b). 